\title{
Serum Theophylline Level as Predictor for Complications in Adults with Acute Theophylline Overdose
}

\author{
Rabab N. Hafez ${ }^{1}$ \\ ${ }^{1}$ Department of Forensic Medicine and Clinical Toxicology, Faculty of Medicine, Ain Shams University, Egypt.
}

All rights reserved.

\begin{abstract}
Acute theophylline overdose is a common medical problem in emergency toxicology departments, it causes serious complications that may lead to death. The Aim of this study is to explore if serum theophylline level is corresponding to development of complications and prolonged hospital stay after acute overdose .Methods: The prospective study included 510 cases admitted to the PCC ASU hospitals during the year 2016.The cases were categorized in two groups; group I(non complicated cases) and group II(complicated cases). All cases were subjected to full history, clinical data and detection of acid base status and ECG performance. Emergency interferences were evaluated. Results: All cases were suicide, 3 cases were died. There was significant difference between groups I and II regarding age, delay time, temperature, vomiting and grade III vomiting, high significant difference regarding hypotension needed IV fluids, very high significant difference regarding pulse, extreme significant difference regarding systolic and diastolic blood pressure, sinus tachycardia, respiratory manifestations and grade I and II vomiting. The frequency of complications during hospital stay was respiratory alkalosis then metabolic acidosis, hematemesis and /or melena, respiratory distress, arrhythmia, agitation, respiratory acidosis and seizures. Regarding ECG Changes in both groups, atrial fibrillation, ventricular tachycardia and cardiac arrest occurred in 3\% of group II. There was extreme significant difference regarding admission to ICU and duration of hospital stay. Activated charcoal, multiple dose activated charcoal and IV fluids were given to all cases of group II with extreme significant difference between both groups regarding multiple dose activated charcoal, gastric lavage and IV fluids. Gastric lavage was done to most of group II cases with extreme significant difference from group I, there was very high significant difference between both groups regarding anticonvulsant administration. There was high significant difference between group I and II regarding peak serum level of theophylline. Regarding relation between serum theophylline level and occurrence of complications, extreme significant difference was found between group I and II regarding metabolic acidosis and hematemesis and/ or melena, very high significant difference regarding arrhythmia and hypotension that needs vasopressor and high significant difference regarding respiratory distress. Regarding relation between peak serum theophylline level and duration of hospital stay, there was high significant difference between the groups for all periods. Conclusion: It was concluded that there was significant difference between the two groups regarding peak serum theophylline level and occurrence of complications and also duration of stay. Recommendations: Serum theophylline level should be considered to be one of the predictors of occurrence of complications, ICU admission and longer duration of stay in acute overdose cases.
\end{abstract}

Key words Acute, Theophylline, Prospective, Overdose.

\section{Introduction}

$\mathrm{T}$ heophylline (1,3-dimethylxanthins) has been used to treat asthma and chronic obstructive pulmonary disease and in neonates it is used for treatment of apnea and bradycardia (Vignola, 2005).

However, in clinical practice, the use of theophylline is limited by its narrow therapeutic range (Ohnishi et al., 2003).

Major mechanisms of theophylline therapeutic efficacy and its toxicity are through the increase of catecholamines release and adenosine antagonism. In addition, high levels of theophylline inhibit phosphodiesterase, resulting in elevation of cyclic adenosine monophosphate and consequent adrenergic stimulation (Hymel 2016).

In overdose, severity ranges from nausea, tremors and headache, to more serious sequelae, such as seizures and life-threatening cardiac dysrhythmias (Barnes, 2005). Seizures are more common with acute overdose than with chronic overdose. In contrast, cardiac dysrhythmias are more common following a chronic overdose rather than acute overdose and with lower serum concentrations (Fisher and Graudins, 2015). 
Serum theophylline level is affected by many conditions increased clearance and consequent low levels are occurred in young age (1-16 years), acidosis, smoking cigarettes and marijuana and concomitant administration of phenytoin, phenobarbital, and rifampin (Blumhof, 2013). In contest, theophylline clearance may be decreased by concomitant diseases prevalent in the elderly and have the potential to increase serum levels. These conditions include impaired renal function, chronic obstructive pulmonary disease, congestive heart failure, hepatic disease and an increased prevalence of use of certain medications with the potential for pharmacokinetic and pharmacodynamic interaction (NIH, 2016).

\section{Aim of the work}

The aim of this study is to investigate if serum theophylline level is correspondance with occurrence of complications and prolonged hospital stay after acute overdose among cases presented to the Poison Control Centre, Ain Shams University Hospitals (PCC-AUH) during the year 2016.

\section{Subjects and Methods}

This study was a prospective study included all patients presented to the PCC, Ain Shams University Hospitals with acute overdose of theophylline from January to December 2016.

- All cases were subjected to full history taking included general characteristics (age, gender, delay time, manner of toxicity, overdose with ordinary or sustained release form)

- Clinical data including vital signs (pulse, blood pressure, temperature, respiratory rate) and full systemic examination.

- Complications during hospital stay including respiratory failure requiring intubation and mechanical ventilation, severe hypotension that need a vasopressor to maintain blood pressure after admission, arrhythmias leading to hemodynamic instability, seizures and status epilepticus

- Criteria related to outcome ICU admission, duration of hospital stay and non survival rate.

- Laboratory analysis:

- Theophylline blood level:

Venous blood samples were withdrawn from each patient on admission and every 6 hours during hospital stay.

The majority of theophylline poisoning cases are due to ingestion of sustained release forms which leads to a prolonged absorption phase and a peak concentration delayed for up to $24 \mathrm{~h}$. For this reason theophylline concentrations should be measured on admission and at 4-6 $\mathrm{h}$ intervals thereafter until theophylline levels are clearly falling (Locas and Donovan, 2013).

Venous blood sample of $2 \mathrm{ml}$ blood was taken after venopuncture of a prominent vein and collected in vacutainer tubes and left for few hours to clot, after complete clotting they were centrifuged.

This was evaluated using Cobas C 311 analyzer manufactured by Roche Company in Germany.

- Arterial blood gases

○ Electrocardiogram (12 leads ECG).
○ Emergency interferences were evaluated including endotracheal intubation, mechanical ventilation and use of dopamine.

\section{Exclusion criteria:}

1. Co-exposure to other drugs which affect cardiovascular function.

2. History of cardiovascular, neurological, renal, hepatic disease and or chronic pulmonary disease.

3. Chronic theophylline toxicity

4. Cases $<18$ years,$>60$ years

5. Concomitant administration of enzyme inducers as phenytoin, phenobarbital, and rifampin or enzyme inhibitors as cimetidine,

6. Smokers

The cases were classified into: Group I: non complicated cases. Group II: complicated cases.

\section{Statistical Analysis:}

The obtained data were revised, coded and organized for statistical analysis using SPSS (Statistical package for Social Science) version (16) software. Data were presented and suitable analysis was done according to the type of data obtained for each parameter (Dahiru, 2008).

Ethical considerations:

Permission was obtained from the Director of the PCCAUH and an ethical approval from Ethical Committee of Faculty of Medicine, Ain Shams University Hospitals. All data were stored anonymously. Valid informed consent was taken from each patient or their guardians.

\section{Results}

Among 23680 cases visited the PCC- AUH during the year 2016, 510 cases $(2.2 \%)$ were diagnosed as acute theophylline poisoning with the inclusion criteria (Table1) All cases were suicide. Regarding age distribution among the two groups, Table 2 showed extreme significant difference.

Regarding gender, $14 \%$ were males and $86 \%$ were females in group I, 9\% were males and $91 \%$ were females in group II with no significant difference (Table 3).

As regards delay time, table 4 showed very high significant difference between both groups.

Comparing group I with group II, vital data showed significant difference regarding temperature, very high significant difference regarding pulse and extreme significant difference between both groups regarding systolic and diastolic blood pressure (Table 5).

Regarding cardiovascular manifestations, table 6 showed that $30 \%$ of group II cases presented with sinus tachycardia, $9 \%$ presented with hypotension needed IV fluids, while $3.8 \%$ of group I cases presented with sinus tachycardia and $0.4 \%$ of cases presented with hypotension needed IV fluids. There was extreme significant difference between group I and II with sinus tachycardia and high significant difference with hypotension needed IV fluids.

Table 7 showed that $45 \%$ of group II cases and no one in group I cases presented with respiratory 
manifestations (tachypnea) with extreme significant difference between the two groups.

Table 8 showed significant difference between group I and II regarding vomiting (77\% and 94\% respectively), while there was no significant difference regarding abdominal colic ( $45 \%$ and $48 \%$ respectively).

As regards grading of vomiting, Table 9 showed extreme significant difference between group I and II regarding grades I and II of vomiting (98.3\% and 3.2\% respectively and $1.4 \%$ and $90.3 \%$ respectively). There was significant difference regarding grade III of vomiting $(0.3 \%$ and $6.5 \%$ respectively).

As regards frequency of complications during hospital stay, table 10 showed that the highest frequency was with respiratory alkalosis $(2.9 \%)$ followed by metabolic acidosis $(2.5 \%)$ then hematemesis and /or melena $(0.8 \%)$ then respiratory distress, arrhythmia ,agitation (0.6\% each). Lastly, respiratory acidosis and seizures ( $0.2 \%$ each).

All cases of group II were admitted to the ICU, while $98.7 \%$ of group I cases were admitted to the Inpatient word and only $1.3 \%$ were admitted to the ICU after being admitted to the Inpatient word. There was extreme significant difference between the two groups (table 11).

Table 12 showed the comparison between the two groups regarding duration of stay; $97.1 \%$ of group I cases were admitted for 1 day, $2.1 \%$ for 2 days, $0.6 \%$ for 3 days and $0.2 \%$ for $>3$ days. $45.5 \%$ of group II cases were admitted for 2 days, $21.2 \%$ for 1 day and for 3 days (each) and $12.2 \%$ for > 3days.There was extreme significant difference between both groups regarding all periods of duration of stay.

Table 13 showed the percentage of outcome of acute theopylline poisoning cases where 3 died and 507 were survived.

Table 14 showed high significant difference between group I and II regarding peak serum level of theophylline.

Table 15 showed distribution of ECG changes in both groups, sinus tachycardia occurred in $30 \%$ of group II

Table 1: Percentage of theophylline poisoned cases in the year 2016

\begin{tabular}{|c|c|c|c|}
\hline Year & No of cases & Acute Theophylline Toxicity cases & $\%$ \\
\hline 2016 & 23680 & 510 & $2.2 \%$ \\
\hline
\end{tabular}

Table (2): Student's t-test comparing group I (non complicated) and group II (complicated) cases as regards age distribution.

\begin{tabular}{|l|l|l|l|l|}
\hline & $\begin{array}{l}\text { Group I } \\
\text { Number }=477\end{array}$ & $\begin{array}{l}\text { Group II } \\
\text { Number = 33 }\end{array}$ & \multirow{2}{*}{$\mathrm{t}$} & \multirow{2}{*}{$\mathrm{P}$-value } \\
\cline { 2 - 4 } & Mean \pm SD & Mean \pm SD & & \\
\hline Age & $21.2 \pm 2$ & $29.6 \pm 8$ & 6.17 & $<0.0001$ \\
\hline
\end{tabular}

SD: Standard Deviation, $P<0.0001$ : extremely significant.

Table (3): Fisher's exact test comparing group I (non complicated) and group II (complicated) group of adult cases as regards gender distribution.

\begin{tabular}{|c|c|c|c|c|c|c|}
\hline \multirow{2}{*}{ Gender } & \multicolumn{2}{|c|}{$\begin{array}{c}\text { Group I } \\
\text { Number }=477\end{array}$} & \multicolumn{2}{c|}{$\begin{array}{c}\text { Group II } \\
\text { Number }=33\end{array}$} & \multicolumn{2}{|c|}{} \\
\cline { 2 - 5 } & Number & $\%$ & Number & $\%$ & F & P-value \\
\hline Male & 68 & 14 & 3 & 9 & \multirow{2}{*}{0.03} & $>0.05$ \\
\hline Female & 409 & 86 & 30 & 91 & & \\
\hline
\end{tabular}

SD: Standard Deviation, P>0.05: Insignificant and in $29 \%$ of group I, atrial fibrillation ventricular tachycardia and cardiac arrest occurred in 3\% (for each).

Activated charcoal, multiple dose activated charcoal and IV fluids were done to all cases of group II and $99.4 \%$, $10 \%$ and $2 \%$ to cases of group I respectively with extreme significant difference between both groups regarding multiple dose activated charcoal and IV fluids, activated charcoal given at the Emergency department. Gastric lavage was done to $66.6 \%$ in group II and $4 \%$ in group I with extreme significant difference between both groups. Anticonvulsant was administrated to $9 \%$ of group II, intubation, vasopressor, nebulizer and mechanical ventilation were administrated to $3 \%$ (for each) of group II. No one in group I received anticonvulsant, intubation, vasopressor, nebulizer or mechanical ventilation with very high significant difference between both groups regarding anticonvulsant use and no significant difference between both groups regarding intubation, vasopressor, nebulizer and mechanical ventilation use (table 16).

Regarding relation between serum theophylline level and occurrence of complications, there was extreme significant difference between group I and II regarding metabolic acidosis and hematemesis and/ or melena , very high significant difference between group I and II regarding arrhythmia and hypotension that needs vasopressor and high significant difference between group I and II regarding respiratory distress. While there was no significant difference between both groups regarding respiratory alkalosis (table 17). There was only one case with respiratory acidosis ,serum theophylline level was $26 \mu \mathrm{g} / \mathrm{ml}$ and one case presented with coma and respiratory distress ,the level was 22 $\mu \mathrm{g} / \mathrm{ml}$ she was died and one case with seizures, the level was $76 \mu \mathrm{g} / \mathrm{ml}$.

Table 18 showed relation between peak serum theophylline level and duration of hospital stay, there was high significant difference between the two groups. while there was no significant difference regarding 
Table (4): Student's t-test comparison between group I (non complicated) and group II (complicated) of cases as regards the delay time.

\begin{tabular}{|l|c|c|c|c|}
\hline & $\begin{array}{c}\text { Group I } \\
\text { Number }=477\end{array}$ & $\begin{array}{c}\text { Group II } \\
\text { Number }=33\end{array}$ & \multirow{2}{*}{$\mathrm{t}$} & \multirow{2}{*}{$\mathrm{P}$-value } \\
\cline { 2 - 4 } & Mean \pm SD & Mean \pm SD & & \\
\hline Delay time(hours) & $7.06 \pm 0.7$ & $7.7 \pm 5.4$ & 3.1 & $<0.001$ \\
\hline
\end{tabular}

SD: Standard Deviation, $P<0.001$ : Very Highly Significant.

Table (5): Student's t-test comparison between group I (non complicated) and group II (complicated) of cases as regards the vital signs.

\begin{tabular}{|c|c|c|c|c|}
\hline \multirow{2}{*}{ Vital data } & $\begin{array}{c}\text { Group I } \\
\text { Number }=477\end{array}$ & $\begin{array}{c}\text { Group II } \\
\text { Number }=33\end{array}$ & \multirow{2}{*}{$\mathrm{t}$} & \multirow{2}{*}{ P -value } \\
\cline { 2 - 4 } & Mean \pm SD & Mean \pm SD & & \\
\hline Temperature $\left({ }^{\circ} \mathrm{C}\right)$ & $37.18 \pm 0.06$ & $37.24 \pm 0.05$ & 2.324 & $\leq 0.05$ \\
\hline Pulse (beats/minute) & $90.8 \pm 5.6$ & $122.7 \pm 18.7$ & 4.890 & $<0.001$ \\
\hline Systolic blood pressure $(\mathrm{mmHg})$ & $121.8 \pm 3.4$ & $87.14 \pm 24.3$ & 36.7 & $<0.0001$ \\
\hline Diastolic blood pressure $(\mathrm{mmHg})$ & $84.5 \pm 6.4$ & $63.7 \pm 8.9$ & 40.813 & $<0.0001$ \\
\hline
\end{tabular}

SD: Standard Deviation, $P<0.0001$ :extremely significant, $P<0.001$ : Very Highly Significant, $P<0.01$ : Highly

Significant, $P \leq 0.05$ : Significant, $P>0.05$ : Insignificant

Table (6): Fisher's exact test comparison between group I (non complicated) and group II (complicated) adult cases as regards cardiovascular manifestations.

\begin{tabular}{|c|c|c|c|c|c|c|}
\hline \multirow{2}{*}{ Cardiovascular manifestations } & \multicolumn{2}{|c|}{$\begin{array}{c}\text { Group I } \\
\text { Number }=477\end{array}$} & \multicolumn{2}{|c|}{$\begin{array}{c}\text { Group II } \\
\text { Number }=33\end{array}$} & \multicolumn{2}{|c|}{} \\
\cline { 2 - 8 } & $\mathrm{N}$ & $\%$ & $\mathrm{~N}$ & $\%$ & $\mathrm{~F}$ & P-value \\
\hline Sinus tachycardia & 18 & 3.8 & 10 & 30 & 0.3 & $<0.0001$ \\
\hline Hypotension treated with IV fluids & 2 & 0.4 & 3 & 9 & 0.5 & $<0.01$ \\
\hline
\end{tabular}

$P<0.0001$ :extremely significant, $P<0.01$ : Highly Significant.

Table (7): Fisher's exact test comparison between group I (non complicated) and group II (complicated) adult cases as regards respiratory manifestations

\begin{tabular}{|c|c|c|c|c|c|c|}
\hline \multirow{2}{*}{ Respiratory manifestations } & \multicolumn{2}{|c|}{$\begin{array}{c}\text { Group I } \\
\text { Number }=477\end{array}$} & \multicolumn{2}{|c|}{$\begin{array}{c}\text { Group II } \\
\text { Number }=33\end{array}$} & \multicolumn{2}{|c|}{} \\
\cline { 2 - 8 } & $\mathrm{N}$ & $\%$ & $\mathrm{~N}$ & $\%$ & $\mathrm{~F}$ & P-value \\
\hline tachypnea & 0 & 0 & 15 & 45 & 0.9 & $<0.0001$ \\
\hline
\end{tabular}

$P<0.0001$ : extremely significant.

Table (8): Fisher's exact test comparison between group I (non complicated) and group II (complicated) adult cases as regards gastrointestinal manifestations.

\begin{tabular}{|c|c|c|c|c|c|c|}
\hline \multirow{2}{*}{ Gastrointestinal manifestations } & \multicolumn{2}{|c|}{$\begin{array}{c}\text { Group I } \\
\text { Number }=477\end{array}$} & \multicolumn{2}{|c|}{$\begin{array}{c}\text { Group II } \\
\text { Number }=33\end{array}$} & \multicolumn{2}{|c|}{} \\
\cline { 2 - 7 } & $\mathrm{N}$ & $\%$ & $\mathrm{~N}$ & $\%$ & $\mathrm{~F}$ & P value \\
\hline vomiting & 369 & 77 & 31 & 94 & 0.06 & $\leq 0.05$ \\
\hline Abdominal colic & 216 & 45 & 16 & 48 & 0.007 & $>0.05$ \\
\hline
\end{tabular}

$P \leq 0.05$ : Significant, $P>0.05$ : Insignificant

Table (9): Fisher's exact test comparison between group I (non complicated) and group II (complicated) adult cases as regards grades of vomiting

\begin{tabular}{|c|c|c|c|c|c|c|}
\hline \multirow{2}{*}{ Grade of vomiting } & \multicolumn{2}{|c|}{$\begin{array}{c}\text { Group I } \\
\text { Number }=369\end{array}$} & \multicolumn{2}{c|}{$\begin{array}{c}\text { Group II } \\
\text { Number }=31\end{array}$} & \multicolumn{2}{|c|}{} \\
\cline { 2 - 8 } & $\mathrm{N}$ & $\%$ & $\mathrm{~N}$ & $\%$ & $\mathrm{~F}$ & P-value \\
\hline Grade I & 363 & 98.3 & 1 & 3.2 & 0.8 & $<0.0001$ \\
\hline Grade II & 5 & 1.4 & 28 & 90.3 & 0.8 & $<0.0001$ \\
\hline Grade III & 1 & 0.3 & 2 & 6.5 & 0.6 & $\leq 0.05$ \\
\hline
\end{tabular}

$P<0.0001$ :extremely significant, $P \leq 0.05$ : Significant

Grades of vomiting according to Deng et al, (2009): grade I once/day, grade II 2-5 episodes /day, may require IV treatment, grade III >6/day may require tube feeding or TPN, grade VI with life threatening complications, grade V fatal. 
Table (10): Frequency of complications during hospital stay in adult cases.

\begin{tabular}{|c|c|c|}
\hline \multirow{2}{*}{ Complications } & \multicolumn{2}{|c|}{} \\
\cline { 2 - 3 } & $\mathrm{N}$ & $\%$ \\
\hline Metabolic acidosis & 13 & 2.5 \\
\hline Respiratory alkalosis & 15 & 2.9 \\
\hline Respiratory distress & 3 & 0.6 \\
\hline Respiratory acidosis & 1 & 0.2 \\
\hline Hematamesis/melena & 4 & 0.8 \\
\hline arrhythmias & 3 & 0.6 \\
\hline agitation & 3 & 0.6 \\
\hline seizures & 1 & 0.2 \\
\hline
\end{tabular}

Table (11): Fisher's exact test comparison between group I (non complicated) and group II (complicated) adult cases as regards place of admission.

\begin{tabular}{|c|c|c|c|c|c|c|}
\hline \multirow{2}{*}{ Place of hospital stay } & \multicolumn{2}{|c|}{$\begin{array}{c}\text { Group I } \\
\text { Number }=477\end{array}$} & \multicolumn{2}{c|}{$\begin{array}{c}\text { Group II } \\
\text { Number = 33 }\end{array}$} & \multicolumn{2}{|c|}{} \\
\cline { 2 - 7 } & $\mathrm{N}$ & $\%$ & $\mathrm{~N}$ & $\%$ & $\mathrm{~F}$ & $\mathrm{P}$-value \\
\hline ICU & 6 & 1.3 & 33 & 100 & 0.8 & $<0.0001$ \\
\hline Inpatient word & 417 & 98.7 & 0 & 0 & & \\
\hline
\end{tabular}

P<0.0001: extremely significant.

Table (12): Fisher's exact test comparison between group I (non complicated) and group II (complicated) adult cases as regards duration of stay.

\begin{tabular}{|l|c|c|c|c|c|c|}
\hline \multirow{2}{*}{ Duration of hospital stay } & \multicolumn{2}{|c|}{$\begin{array}{c}\text { Group I } \\
\text { Number }=477\end{array}$} & \multicolumn{2}{c|}{$\begin{array}{c}\text { Group II } \\
\text { Number }=33\end{array}$} & \multicolumn{2}{|c|}{} \\
\cline { 2 - 7 } & $\mathrm{N}$ & $\%$ & $\mathrm{~N}$ & $\%$ & $\mathrm{~F}$ & $\mathrm{P}$-value \\
\hline - 1 day & 463 & 97.1 & 7 & 21.2 & 0.6 & $<0.0001$ \\
$\bullet \quad$ 2 days & 10 & 2.1 & 15 & 45.5 & 0.5 & $<0.0001$ \\
$-\quad 3$ days & 3 & 0.6 & 7 & 21.2 & 0.6 & $<0.0001$ \\
$\bullet \quad>$ 3 days & 1 & 0.2 & 4 & 12.2 & 0.7 & $<0.0001$ \\
\hline
\end{tabular}

$P<0.0001$ : extremely significant.

Table (13): Percentage of outcome of the theophylline poisoned cases

\begin{tabular}{|l|l|l|}
\hline Outcome & No of cases & $\%$ \\
\hline Survived & 507 & $99.4 \%$ \\
\hline Deaths & 3 & $0.6 \%$ \\
\hline Total & 510 & 100 \\
\hline
\end{tabular}

Table (14): Student's t-test comparison between group I (non complicated) and group II (complicated) cases as regards peak serum theophylline level.

\begin{tabular}{|l|c|c|c|c|}
\hline \multirow{2}{*}{ Theophylline level } & $\begin{array}{c}\text { Group I } \\
\text { Number = 477 }\end{array}$ & $\begin{array}{c}\text { Group II } \\
\text { Number = 33 }\end{array}$ & \multirow{2}{*}{$\mathrm{t}$} & \multirow{2}{*}{ P value } \\
\cline { 2 - 3 } & Mean \pm SD & Mean \pm SD & & \\
\hline Peak level $(\mu \mathrm{g} / \mathrm{ml})$ & $24.14 \pm 13.9$ & $32.85 \pm 20.4$ & 2.754 & $<0.01$ \\
\hline
\end{tabular}

SD: Standard Deviation, $P<0.01$ : Highly Significant.

Table (15): Percentage of distribution of ECG Changes in group I (non complicated) and group II (complicated) adult cases.

\begin{tabular}{|c|cc|cc|}
\hline \multirow{2}{*}{ ECG Changes } & \multicolumn{2}{|c|}{$\begin{array}{c}\text { Group I } \\
\text { Number }=477\end{array}$} & \multicolumn{2}{c|}{$\begin{array}{c}\text { Group II } \\
\text { Number }=33\end{array}$} \\
\cline { 2 - 5 } & no & $\%$ & no & $\%$ \\
\hline Sinus tachycardia & 138 & 29 & 10 & 30 \\
\hline AF/ SVT & 0 & 0 & 1 & 3 \\
\hline V Tach & 0 & 0 & 1 & 3 \\
\hline VF/ Arrest & 0 & 0 & 1 & 3 \\
\hline
\end{tabular}

AF: atrial fibrillation, SVT: supraventricular tachycardia, V Tach: ventricular tachycardia, VF: ventricular fibrillation. 
Table (16): Fisher's exact test comparison between group I (non complicated) and group II (complicated) adult cases as regards management.

\begin{tabular}{|c|c|c|c|c|c|c|}
\hline \multirow[b]{2}{*}{ Management } & \multicolumn{2}{|c|}{$\begin{array}{l}\text { Group I } \\
\text { Number }=477\end{array}$} & \multicolumn{2}{|c|}{$\begin{array}{l}\text { Group II } \\
\text { Number }=33\end{array}$} & \multirow[b]{2}{*}{$\mathrm{F}$} & \multirow[b]{2}{*}{$\mathrm{P}$-value } \\
\hline & $\mathrm{N}$ & $\%$ & $\mathrm{~N}$ & $\%$ & & \\
\hline $\begin{array}{ll}\text { Decontamination } \\
\text { - } \\
\text { - } \quad \text { Activated Charcoal } \\
\text { - Multiple dose Activated Charcoal }\end{array}$ & $\begin{array}{l}20 \\
474 \\
48\end{array}$ & $\begin{array}{l}4 \\
99.4 \\
10\end{array}$ & $\begin{array}{l}22 \\
33 \\
33\end{array}$ & $\begin{array}{l}66.6 \\
100 \\
100\end{array}$ & $\begin{array}{l}0 \\
0.06 \\
0.4\end{array}$ & $\begin{array}{l}<0.0001 \\
>0.05 \\
<0.0001\end{array}$ \\
\hline $\begin{array}{ll}\text { Supportive treatment } \\
\text { - } \\
\text { - } \text { Anticonvulsaids } \\
\text { - Intubation } \\
\text { - Vasopressor } \\
\text { - Nebulizer } \\
\text { - Mechanical ventilation }\end{array}$ & $\begin{array}{l}12 \\
0 \\
0 \\
0 \\
0 \\
0\end{array}$ & $\begin{array}{l}2 \\
0 \\
0 \\
0 \\
0 \\
0\end{array}$ & $\begin{array}{l}33 \\
3 \\
1 \\
1 \\
1 \\
1\end{array}$ & $\begin{array}{l}100 \\
9 \\
3 \\
3 \\
3 \\
3\end{array}$ & $\begin{array}{l}0.7 \\
0.9 \\
0.9 \\
0.9 \\
0.9 \\
0.9\end{array}$ & $\begin{array}{l}<0.0001 \\
<0.001 \\
<0.01 \\
<0.01 \\
<0.01 \\
<0.01\end{array}$ \\
\hline
\end{tabular}

$P<0.0001$ :extremely significant, $P<0.001$ : Very Highly Significant, $P<0.01$ : Highly Significant, $P \leq 0.05$ : Significant, $P>0.05$ : Insignificant

Table (17): Student's t-test relation between peak serum theophylline level and complications.

\begin{tabular}{|c|c|c|c|c|}
\hline & \multicolumn{2}{|c|}{ Peak serum theophylline level $(\mu \mathrm{g} / \mathrm{ml})$} & \multicolumn{2}{|l|}{ t-Test } \\
\hline & Non complicated cases & Complicated cases & \multirow[b]{2}{*}{$\mathrm{t}$} & \multirow{2}{*}{$\mathrm{P}$-value } \\
\hline & Mean \pm SD & Mean \pm SD & & \\
\hline Arrhythmias & $19 \pm 12.3$ & $51.7 \pm 32.9$ & 3.761 & $<0.001$ \\
\hline Hypotension needs vasopressor & $8.375 \pm 2.326$ & $19 \pm 4.583$ & 5.2 & $<0.001$ \\
\hline Metabolic acidosis & $24.32 \pm 14.3$ & $57.46 \pm 21.5$ & 6 & $<0.0001$ \\
\hline Respiratory alkalosis & $22.55 \pm 17.38$ & $38.3 \pm 26.35$ & 1.473 & $>0.05$ \\
\hline Respiratory distress & $22.7 \pm 13.2$ & $45.3 \pm 45$ & 2.89 & $<0.01$ \\
\hline Hematamesis/melena & $13.58 \pm 6.5$ & $55.75 \pm 23.37$ & 6.8 & $<0.0001$ \\
\hline
\end{tabular}

SD: Standard Deviation, $P<0.0001$ : extremely significant, $P<0.001$ : Very Highly Significant, $P<0.01$ : Highly Significant, $P \leq 0.05$ : Significant, $P>0.05$ : Insignificant

Table (18): ANOVA relation between peak serum theophylline level of cases and duration of hospital stay

\begin{tabular}{|l|l|l|l|l|l|}
\hline \multirow{2}{*}{ Peak $(\mu \mathrm{g} / \mathrm{ml})$} & \multicolumn{4}{|l|}{ duration of hospital stay } & \\
\cline { 2 - 6 } & 1 day & 2 days & 3 days & $>3$ days & P-value \\
\hline Mean \pm SD & 13.4 & 36.6 & 37.2 & 71.14 & $<0.01$ \\
& \pm 4.9 & \pm 20.8 & \pm 34.4 & \pm 38.18 & \\
\hline
\end{tabular}

SD: Standard Deviation, $P<0.01$ : Highly Significant.

\section{Discussion}

Acute theophylline overdose is a toxicological emergency especially complicated cases who have high morbidity (Hymel, 2016). This study revealed that during the year 2016,510 cases $(2.2 \%)$ were diagnosed as acute theophylline overdose. Hocaoglu et al.,(2014) reported that between 1993 and 2011; 354 (0.4\%) of cases were due to acute theophylline exposure. This study revealed also that all cases were suicidal attempts. This agrees with Olson, (2012) who stated that acute overdose is usually a result of a suicide attempt, also, Hocaoglu et al., (2014) reported that intentional exposure was significantly higher due to high tendency to take large dose at the time of committing suicide.

The current study showed that the mean age in the complicated group was younger than that of the noncomplicated group with extreme significant difference. Two of died cases were above 50 years old as the clearance of theophylline is decreased by increasing age (Ohnishi et al., 2003). Also, theophylline clearance may be further reduced by concomitant diseases developed with increasing the age, which further impair clearance of this drug and have the potential to increase serum levels and potential toxicity. In addition to an increased prevalence of use of certain medications with the potential for pharmacokinetic and pharmacodynamic interaction. Protein binding may be decreased by increasing the age resulting in an increased proportion of the serum theophylline concentration in the pharmacologically active unbound form (Fisher and Graudins, 2015).

This study showed that there was no significant difference between the two groups regarding gender. Fisher and Graudins, (2016) reported that gender differences in theophylline clearance are relatively small and unlikely to be of clinical significance. At the same time it was more prevalent in females in both groups as intentional poisoning is more common in females as was reported by Hocaoglu et al. (2014).

There was very high significant difference between the two groups regarding delay time in the current study, increasing delay time is directly proportional to severity of toxicity (Mowry et al., 2015). 
The current study showed significant difference between both groups regarding temperature, very high significant difference regarding pulse and extreme significant difference regarding systolic and diastolic blood pressure. In addition, sinus tachycardia was present in most of group II cases and only in 3.8\% only with of group I cases presented with extreme significant difference between both groups. Hypotension required treatment with IV fluids was present in $9 \%$ of group II cases and $0.4 \%$ of group I cases with high significant difference. Olson, (2012) found that tachycardia and hypotension occur frequently with acute toxic overdoses of theophylline. Hymel, (2016) reported that hypotension occurs with severe overdoses. Bethesda, (2016) stated that theophylline stimulates the vasomotor and vagal centers promotes vasoconstriction and bradycardia, respectively, but the overall effect of theophylline on heart rate and blood pressure depends on whether CNS or peripheral effects predominate.

This study revealed that there was extreme significant difference between the two groups regarding tachypnea. Bateman et al. (2014) reported that acute theophylline toxicity causes stimulation of respiratory center leading to hyperventilation and respiratory alkalosis and in severe cases respiratory failure and arrest may occur.

The current study showed that there was significant difference between the two groups regarding occurrence of vomiting and most of group II cases that complained of vomiting were of grade II, while most of group I cases were of grade I. There was no significant difference regarding abdominal colic. Bateman et al. (2014) mentioned that vomiting occurs very common in acute theophylline cases $(75 \%)$ it is pronounced and resistant to antiemetic treatment, in protracted vomiting associated hematemesis may occur and abdominal pain may also accompany vomiting. Bethesda, (2016) explained by stating that theophylline toxicity causes increase in gastric acid secretion with delay in emptying ,it may stimulate the vomiting center leading to severe repeated vomiting and gastric erosions regarding complications, the present study revealed that the highest frequency was with respiratory alkalosis $(2.9 \%)$ followed by metabolic acidosis $(2.5 \%)$ then hematemesis and /or melena $(0.8 \%)$ then respiratory distress, arrhythmia, agitation $(0.6 \%)$ each. Lastly, respiratory acidosis and seizures $(0.2 \%)$ each. Theophylline causes stimulation of the medullary respiratory center directly and indirectly through constriction of cerebral vasculature with the resultant decrease in cerebral blood flow and increase in carbon dioxide tension causing increase depth and rate of respiration with subsequent respiratory alkalosis also, it causes agitation and seizures which is a severe complication and frequently resistant to anticonvulsant drugs (Bethesda, 2016). Metabolic acidosis occurs in severe cases due to multisystem organ failure (Olson, 2012).

Arrhythmia occurs in severe cases secondary to increased secretion of catecholamines (Hymel, 2016). Respiratory acidosis occurs due to CNS depression in severe toxicity (Fisher and Graudins, 2015).
The current study showed that there was extreme significant difference between the two groups regarding ICU admission as all group II cases were admitted to ICU, while only $1.3 \%$ of group I cases were transferred to ICU during hospital stay due to development of complications as hypotension and severe vomiting with GIT bleeding. Complicated cases as cardiac dysrythmias, severe hypotension, coma, seisures, acid base disturbance or GIT bleeding must be admitted to ICU (Jolliff et al., 2013).

This study revealed that there was extreme significant difference between the two groups regarding duration of stay; most of group II cases were admitted for 2 days (45.5\%), 21.2\% were admitted for 3 days, 12.2 for more than 3 days. While most of group I cases were admitted for one day $(97.1 \%)$ and minority of cases were admitted for 3 days or more $(0.6 \%)$ and $(0.2 \%)$ respectively. Cases admitted to ICU need longer duration for hospital stay as they need close observation and medical care for proper treatment of complications (Hocaoglu et al., 2014).

In the present study, out of 510 cases, 3 cases were died due to respiratory distress required mechanical ventilation, one of them was complicated with ventricular fibrillation and developed cardiac arrest. Hocaoglu et al., (2014) found that out of 354 cases one case was died. Acute theopylline toxicity may lead to acute lung injury (ALI) and respiratory failure leads to arrest (Hymel, 2016). In addition, theophylline stimulates all levels of the CNS including respiratory center stimulation which may end in respiratory failure (Bethesda, 2016).

Other causes of death were described by Rumack, (2017) who found that severe and fatal toxicities result from seizures, rhabdomyolysis, hypotension and cardiac dysrhythmias. Seizures may occur even without other preceding symptoms of toxicity and often result in death.

Regarding ECG changes, the present study showed that sinus tachycardia occurred in $30 \%$ of group II and in $29 \%$ of group I, atrial fibrillation occurred in $3 \%$ of group II. Ventricular tachycardia occurred in $3 \%$ of group II and cardiac arrest occurred in $3 \%$ of group II. Hymel (2016) found that the commonest arrhythmia is sinus tachycardia, other forms which may occur are atrial fibrillation, atrial flutter, supraventricular tachycardia, multifocal atrial tachycardia, ventricular tachycardia, ventricular fibrillation and cardiac arrest.

All cases of group II received activated charcoal at the emergency room then multiple dose activated charcoal, all of them needed IV fluids as supportive treatment $(9 \%$ of them for correction of hypotension), most of them needed gastric lavage $(66.6 \%)$ with extreme significant difference with group I cases regarding the previous methods of treatment except activated charcoal as there was no significant difference; most of group I cases received activated charcoal at the emergency room $(99.4 \%), 10 \%$ needed multiple dose activated charcoal, $4 \%$ needed gastric lavage and $2 \%$ needed IV fluids for correction of hypotansion and severe vomiting with GIT bleeding. On the other hand, $9 \%$ of group II cases needed anticonvulsant to control agitation and seizures. In the 
present study, only one case needed hemodialysis, his theophylline level was $98 \mu \mathrm{g} / \mathrm{ml}$ and complicated by metabolic acidosis.

Management decisions should be based on both clinical assessment and theophylline concentrations (Hymel, 2016). In cases of hypotension, IV access fluids often give a good response, if hypotension is refractory to fluids a noradrenaline infusion should be administrated with continuous ECG monitoring. Tachydysrhythmias should be treated with IV cardioselective beta blockers. IV metoprolol $5 \mathrm{mg}$ slowly, a repeat dose of $5 \mathrm{mg}$ IV can be given after 5 minutes if the initial response is inadequate, endotracheal intubation may be needed in patients who require high-dose benzodiazepines or barbiturates to control seizures (Hayes, 2011).

Hocaoglu et al., (2014) reported that $83 \%$ of cases needed gastrointestinal decontamination. Consider gastric lavage (unless contraindicated) if the patient has recently $(<1 \mathrm{~h})$ ingested a significant amount or a sustained-release preparation of theophylline or if theophylline bezoar formation is suspected after insertion of an endotracheal tube. Activated charcoal enhances elimination of theophylline. It is a very effective method of elimination. Multiple dose activated charcoal (MDAC) is considered the mainstay treatment of theophylline toxicity in severe cases. It is important to aggressively control nausea and vomiting in order to perform MDAC treatment. It is also important that the patient is able to protect his or her airway in order to prevent aspiration of activated charcoal. Administer the cathartic, sorbitol, with the activated charcoal one time (Antoniou et al., 2010). Hemodialysis is the definitive lifesaving procedure in severe toxicity and is highly effective if initiated early. Arrangements for hemodialysis should therefore be made early when life-threatening severe toxicity has occurred or is anticipated, patients at risk of death should be identified and dialyzed before clinical deterioration occurs as serum theophylline level $>80 \mu \mathrm{g} / \mathrm{ml}$ in the setting of acute overdose, clinical manifestations of severe toxicity such as arrhythmias, (including SVT), hypotension or seizures (Hayes, 2011)

However Savory and Wills (2012) found that there is discrepancy between toxic theophylline levels and symptoms in acute compared to chronic overdose. Also, Rumack (2017) found that serum concentrations do not always accurately predict acute toxicity, the current study revealed high significant difference between group I and II regarding serum theophylline level indicating the relation between high serum level and presence of complications as it was revealed from the extreme significant difference regarding the presence or non-presence of metabolic acidosis and hematamesis and/or melena, very high significant difference regarding hypotension needing vasopressor and arrhythmia, high significant difference regarding respiratory distress, while there was no significant difference regarding respiratory alkalosis.

Theophylline concentrations gives diagnostic and prognostic information, define the need for and measure the efficacy of therapy in overdose. Theophylline concentrations predict the risk of seizures and cardiac toxicity in acute overdose (Dawson and Whyte, 1999). Blumhof (2013) and Sessler (1990) found relation between serum theophylline level and presence of complications. In adult patients with acute ingestions the incidence of seizures and arrhythmias is increased when the serum concentrations approaching $80 \quad \mu \mathrm{g} \quad / \mathrm{mL}$. However, as there is often a lag time between achieving potentially toxic concentrations and having a major complication it has been suggested that these concentrations should be used as an indication for extracorporal removal (charcoal haemoperfusion or haemodialysis) (Mowry et al.,2015)

The present study showed high significant difference between different durations of hospital stay according to serum theophylline level, higher serum theophylline levels are associated with longer duration of stay mostly due to presence of complications at these levels.

\section{Conclusion}

It was concluded that there was significant difference between the two groups regarding peak serum theophylline level and occurrence of complications and also duration of stay.

\section{Recommendations}

Serum theophylline level should be considered to be one of the predictors of occurrence of complications, ICU admission and longer duration of stay in cases of acute theophylline overdose.

\section{References}

Antoniou T, Gomes T, Mamdani M,et al., (2010): Ciprofloxacine induced theophylline toxicity: a population based study. European Journal of Clinicl Pharmacology; 67(5) p521-526

Barnes P (2005) Theophylline in chronic obstructive pulmonary disease: New Horizons. Proc Am Thorac Soc 2:334-339.

Bateman N, Jefferson R, Thomas S et al., (2014): Theophylline: Oxford Desk Reference Toxicology; $1^{\text {st }}$ ed.p146-147.Oxxord University Press ,UK.

Bethesda, (2016): Drug information. American Society of Health System Pharmacists p 3606.

Blumhof S (2013): Theophylline Level. http://emedicine.medscape.com/article/209039 5-overview\#a2 Dec 19.

Dahiru T. (2008): P-value, a true test of statistical significance? A cautionary note. Annals of Ibadan Postgraduate Medicine 6: 21-26.

Dawson A and Whyte I (1999): Therapeutic Drug monitoring in drug overdose . Br $\mathrm{J}$ Clinical Toxicol Sep; 48(3): 278-283.

Deng E, Frankel M, Cohen L et al.,2009 Evidence based clinical practice guidelines for intergrative oncology. complimentary therapies and botanicals.J Soc Inter Oncol. 2009; 7(3):169-76

Hayes J, Frank D, Murray L et al.,(2011):Theophylline toxicity. in Toxicology Handbook $2^{\text {nd }}$ ed 2011. P 348-361

Fisher J and Graudins A (2015). Intermittent haemodialysis and sustained low-efficiency dialysis (SLED) for acute theophylline toxicity. J Med Toxicol. Sep. 11 (3):359-6 
Fisher J and Graudins A (2016): DailyMed. Current information for theophylline (Anhydrous) tablet, extended release. July 21, https://dailymed.nlm.nih.gov/dailymed/drugInf o.cfm?setid=038c2b07-8028-4dc4-847cadafe1b0e81a

Hocaoglu N, Yildiztepe E, Bayram B et al., (2014): Demographic and clinical characteristics of theophylline exposures between 1993-2011. Balkan Med J 31 (4): 322-7

Hymel G (2016): Patients older than 60 years or younger than 3 years. Theophylline Toxicity Treatment \& Management Jan 07, article 818847 midescape

Jolliff H, Fletcher E, Roberts K, et al. (2013): Pediatric hydrocarbon-related injuries in the United States: 2000-2009. Pediatrics; 131:11391147

Lucas C, Donovan P (2013): Just a repeat - When drug monitoring is indicated. Aust Fam Phycisino. Jan-Feb;42(1-2):18-22.

NIH (2016): Daily Med. Current Medication Information for Theophylline (Anhydrous) Tablet, Extended Release. July 21, https://dailymed.nlm.nih.gov/dailymed/drugInf o.cfm?setid=038c2b07-8028-4dc4-847cadafe1b0e81a.

Mowry JB, Spyker DA, Brooks DE, et al., (2015): Annual Report of the American Association of
Poison Control Centers' National Poison Data System (NPDS): 32nd Annual Report. Clin Toxicol (Phila). Dec. 53 (10):962-1147

Ohnishi A, KatoM, Kojima J,et al.,(2003): Differential Pharmacokinetics of theophylline in elderly patients .Drugs Aging ; 20 (1): 71-84.

Olson K. (2012). Poisoning and Drug Overdose, Sixth Edition. McGraw-Hill, New York, NY, p. 386-7

Rumack B, (2017): Information System Micromedex, Inc., Englewood, CO, 2017; CCIS Volume 172, edition expires May. Hall AH \& Rumack BH (Eds): TOMES(R) Information System Micromedex, Inc., Englewood, CO, 2017; CCIS Volume 172, edition expires May, 2017.

Savory J and Wills M ,(2012):Biological monitoring of exposure to toxic trace elements.Clinical Chemistry : An Overview p 107-109.

Sessler C,(1990):Theophylline toxicity:clinical featuresof 116 consecutive cases. Am J Med 88 (6): 567-76 (1990)

Vignola A(2004) : PDE4 inhibitors in COPDFa more selective

approach to treatment. Instituto di Biomedicina e Immunologia Molecolare, Consiglio Nazionale delle Ricerche; Ospedale V. Cervello, IT90146, Palermo, Italy, Respiratory Medicine : 98, 495-503

\title{
الملخص العربي - n
}

\section{مستوى الثيوفللين فى المصل كمتنبا لحدوث المضاعفات فى البالغين المتعرضين للزياده الحاده لجرعه الثيوفللين}

\author{
رباب نبيل حافظ
}

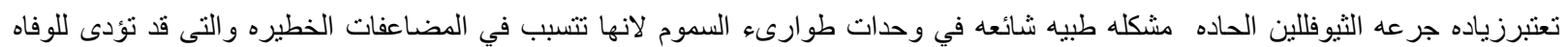

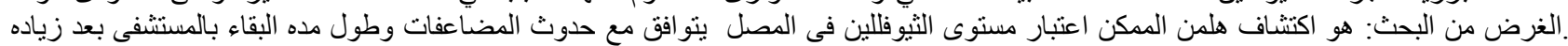

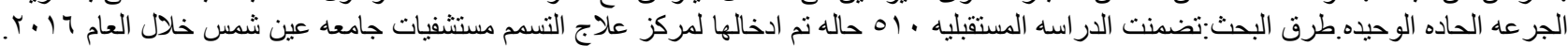

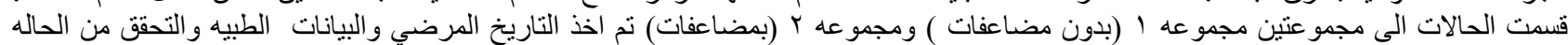

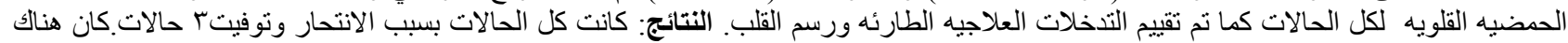

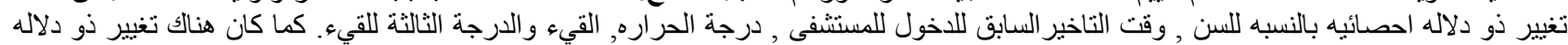

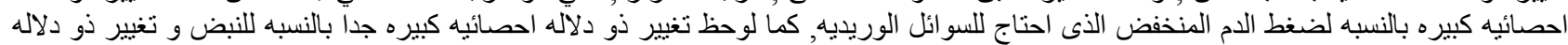

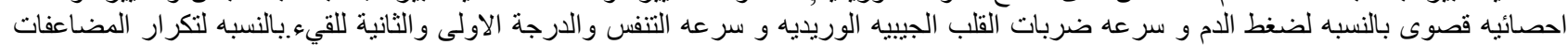

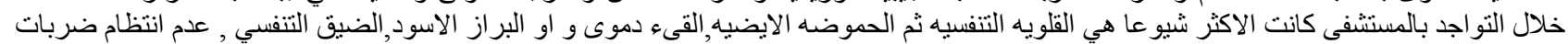

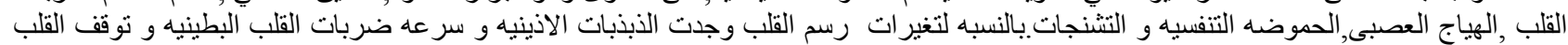

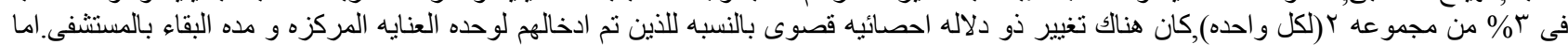

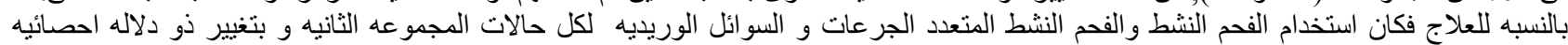

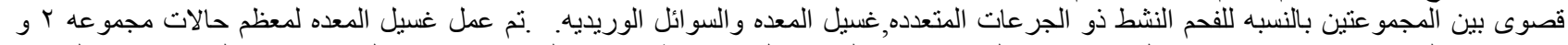

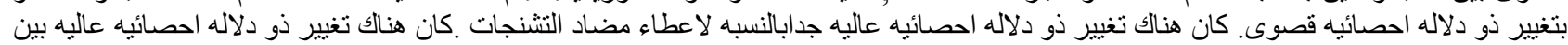

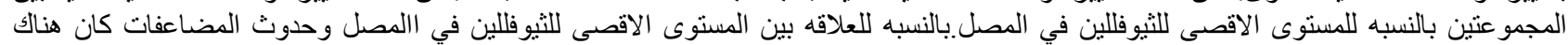

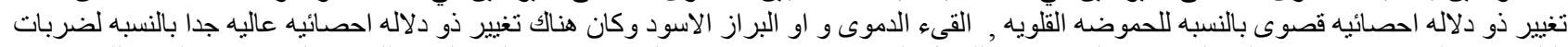

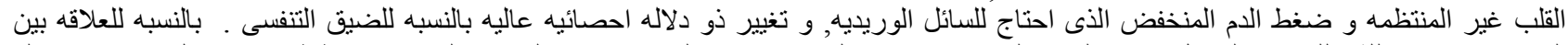

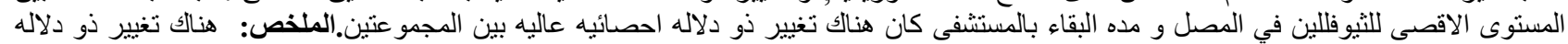

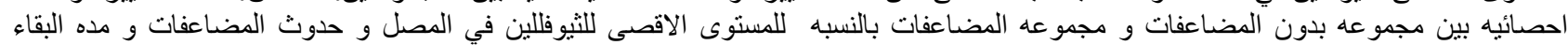

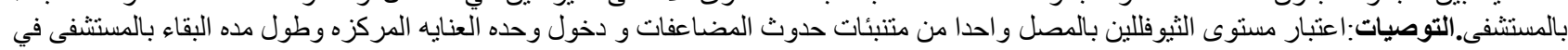

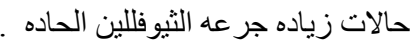

